\title{
Very young neutron stars and millisecond pulsars: the role of the accretion
}

\author{
Alexander F. Kholtygin and Andrei P. Igoshev \\ Institut of Astronomy, Saint Petersburg State University, Russia \\ email: afkholtygin@gmail.com; ignotur@gmail.com
}

\begin{abstract}
We consider the evolution of the very young neutron stars (NS) with moderate and low magnetic field values around $1 \mathrm{E} 8 \mathrm{G}$ to know how large is the share of the these objects among the those attributed as the millisecond pulsars (MSP). To exclude the contamination of accreted NS and young NS with moderate magnetic fields we study the observational evidences of the accretion on NS in the binary systems and different methods of age determinations. It was concluded that only central compact objects are appropriate candidates for NSs with small initial magnetic fields.
\end{abstract}

Keywords. Stars: Neutron, pulsars: general, magnetic fields, accretion

\section{Millisecond pulsars}

Very young neutron stars are younger then a few Kyear. The method of age determination might be divided into two distinctive groups. First group contains age determinations based on evolutionary properties of stars such as the spin-down age and spectrum. These methods demand accurate theoretical models describing evolution of NSs. The second group includes methods based on kinematic of neutron stars. These are such methods as determination of distance from the plane of the Milky Way, integration of pulsars paths back in time or an analysis of the distance from associated SN remnant. The methods of the first group are verified by methods from the second group.

It is known that millisecond pulsars are extremely old. This estimate is confirmed by their spin-down age. All millisecond pulsars show clear $P(\dot{P})$ dependance with naturally explained dispersion. In fact, disc accretion introduces an additional connection between $P$ and $\dot{P}$ in form (Alpar et al. 1982):

$$
\dot{P} \sim 3.2 \cdot 10^{-20} I_{45}^{-1}\left(10^{3} P\right)^{4 / 3}\left(\frac{M}{M_{\odot}}\right)^{5 / 3} \dot{m}_{17}
$$

Here $P$ is period of pulsar in seconds, $I_{45}$ is the neutron star moment of inertia in units of $10^{45} \mathrm{~g} \mathrm{~cm}^{2}, M$ is mass of NS and $\dot{m}_{17}$ is the accretion rate in units of $10^{17} \mathrm{~g} \mathrm{~s}^{-1}$. As soon as the accretion stage is finished, NS begins braking due to standard electromagnetic dipole radiation shifting to the right side of $P \dot{P}$ plane along of constant magnetic field line. Therefore, only pulsars with $P$ much smaller than that predicted by the accretion model are under suspicion that they are in reality the very young NS. There are two of them: B1820-30A and B1821-24A both are located in globular clusters.

Then we conclude that it is impossible to distinguish young neutron stars with initial small magnetic field from MSP. Not only the small amount of exclusions, but also uncertainty of their radial velocities prevent us from conclusion. Also spectrum of X-ray radiation Zavlin (2007), Zavlin (2009) even for isolated MSP show their antiquity. Therefore, all millisecond pulsars should be excluded from objects with small initial magnetic field. 


\section{Central compact objects}

However, ordinary and millisecond pulsars are not all NS, they outline areas where it is possible to emit non-thermal electromagnetic radiation. Nevertheless, modern astrophysics notes also NSs with unusual for pulsars properties which could probable help us to solve this puzzle. Nowadays, we observe dim isolated neutron stars (DINs) and central compact objects (CCO). CCO are definitely the youngest NSs because of their connections with SN remnant and thermal X-ray emission. DINs are thoughts to be ordinary young pulsars which are not observable due to bad beaming. CCO are deprived from any manifestations of magnetic fields (while the Crab pulsar has similar age). CCO have pure thermal X-ray spectrum and no variability Pavlov et al. (2004).

The nature of CCO is still enigmatic. No reliable determination of $P$ is available for them. It is possible to suggest that CCO have really small magnetic fields or longer period (lies beyond the pulsar death-line). Strong magnetic field leads to appearance of inhomogeneity of surface temperature both due to bombarding of poles by accelerated particles and different crust conductivity. Therefore, small initial magnetic fields are preferable. Despite CCO high temperature, thermal instability apparently does not work Blandford et al. (1983).

\section{Conclusion}

We remark that current observations suggest only 6 objects which should be treated as NSs with small or missing magnetic fields. Based on the birthrate of visible pulsars we should conclude that at once $20 \%$ of all NS should be born as CCO. However, we does not observe $\mathrm{CCO}$ with ages more than $2 \cdot 10^{4}$ years. Future investigation should show if $\mathrm{CCO}$ go through significant growth of surface magnetic fields or fast cooling.

\section{References}

Pavlov, G. G., Divas, S., \& Marcus, A. T. 2004, IAU Symposium, 218, 239

Alpar, M. A., Cheng, A. F., Ruderman, M. A., \& Shabam, J. 1982, Letters to Nature, 30, 728

Zavlin, V. E. 2007 ApSS, 308, 1-4, 297

Zavlin, V. E. 2009 Neutron Stars and pulsars, Astronomy and Space Science Library, 357, 181

Blandford, R. D., Applegate, J. H., \& Hernquist, L. 1983 MNRAS, 204, 1025 